Background: Body mass and cognitive status are independently associated with physical and functional outcomes in older adults. However, accumulating evidence suggests that higher body mass may be protective during late life, in late life. Yet little is known regarding the impact of cognitive impairment at various levels of body mass in older adults.

Methods: We examined prevalence and odds of physical and IADL limitations by cognitive status (normal/impaired) and body mass category (normal, overweight, obese) in 4,033 older men and 5,563 women aged 60+ from 2006/ 2008 waves of Health and Retirement Study. Models were adjusted for age, race, education, physical activity level, comorbidity and smoking status. Individuals with TICS score $\geq 7$ were classified as cognitively intact/normal (REF); TICS scores $<7$ were classified as cognitively impaired. Physical limitation (PL) was determined using the HRS summary score; IADL limitation was defined as difficulty/inability with meals, chores, managing money. Models were adjusted for age, race, education, physical activity level, comorbidity and smoking status and weighted.

Results: Prevalence of PL in adults with cognitive impairment in men were $50.7 \%, 52.3 \%$ and $67.3 \%$ for normal, overweight and obese categories; for women, rates of PL were $61.8 \%, 71.9 \%, 81.8 \%$. Among men, cognitive impairment was significantly associated with higher rates of physical impairment in normal OR 2.11 (1.07-4.21), overweight OR 2.17 (1.17-4.03) and obese OR 1.09 (0.59-2.01weight categories. Among women, adults who were cognitive impairment was associated with physical limitations in normal OR 2.11 (1.07-4.21) and overweight categories OR 1.71 (1.0-2.91) but not obese OR 1.09 (0.59-2.01). Association between obesity and cognition on IADL limitations are also discussed.

Conclusion: Older adults with poor cognition are at increase odds of physical limitations independent of body mass. The impact of cognitive impairment in combination with obesity varies between men and women and merits further investigation.

\section{METABOLIC SYNDROME AND DISABILITY IN CHINESE NONAGENARIANS AND CENTENARIANS}

M. Yang, B. Dong, Q. Hao, West China Hospital, Sichuan University, Chengdu, Sichuan, China

Metabolic syndrome (MetS) increases with aging. Little is known about the relationship between MetS and disability in the elderly population, especially in the oldest old. We therefore conducted this study to investigate the possible association between MetS and disability in a population of Chinese nonagenarians and centenarians. Data were obtained from a face-to-face survey conducted in 870 Chinese adults aged 90 years or older. MetS was diagnosed according to the International Diabetes Federation criteria. Activities of daily living (ADL) and instrumental activities of daily living (IADL) disabilities were evaluated using the physical self-maintenance scale and the Lawton-Brody IADL scale, respectively. The subjects included in the current analyses were 500 women and 225 men (mean age: $93.8 \pm 3.1$ years). MetS was present $13.0 \%$ in women and $9.8 \%$ in men. In women, the prevalence of ADL and IADL disabilities was significantly higher in the MetS group compared with the non-MetS group (ADL:43.1\% vs. $30.6 \%, \mathrm{p}=0.044$; IADL:
$73.8 \%$ vs. $59.8 \%, \mathrm{p}=0.030$ ). After adjusting for relevant confounders, the MetS group showed significantly increased odds ratios (ORs) for either ADL $1.8,95 \%$ confidence interval [CI] 1.2-3.4) or IADL disability (2.1, 95\% CI 1.3-4.7) compared with the non-MetS group. In men, similar results were found with respect to the prevalence of ADL or IADL disability and adjusted ORs between MetS and non-MetS groups, but these results were not statistically significant. In conclusion, in our study population, MetS appeared to be associated with an increased risk of either ADL or IADL disability, especially in women.

\section{PLANNING HEALTH SERVICES FOR SENIORS: CAN WE USE PATIENTS' OWN PERCEPTION?}

S. Figueiredo ${ }^{1,2}$, A. Rosenzveig ${ }^{2}$, J.A. Morais ${ }^{3,4}$, N.E. Mayo ${ }^{5,1,4}, 1$. School of Physical and Occupational Therapy, McGill University, Montreal, Quebec, Canada, 2. Center for Outcomes Research and Evaluation, Research Institute, McGill University Health Centre, Montreal, Quebec, Canada, 3. Faculty of Medicine, McGill University, Montreal, Quebec, Canada, 4. Department of Geriatrics, McGill University Health Centre, Montreal, Quebec, Canada, 5. Division of Clinical Epidemiology, McGill University, Montreal, Quebec, Canada

Across Canada, people over 75 years of age represent $16 \%$ of all hospital admissions. The admission rate per 100,000 seniors is 5 times higher for acute care and 22 times higher for complex continuing care than the rates for younger adults. After a hospital discharge, dealing with new disabilities can be difficult and even overwhelming. Understanding patients' needs in a timely manner may allow services to be allocated to those at highest risk for deterioration, thus, improving care while optimizing health care cost. Could patient's perceptions of how they are feeling be used as a marker of potential need for post-discharge services?

The aim of the study was to estimate whether selfreported health can be used as an indicator of service needs among seniors.

In this cross-sectional survey, age and sex-adjusted logistic regression was used to estimate the link between functional status indicators and fair or poor self-reported health. Results were reported as Odds Ratio (OR) and its $95 \%$ confidence interval $(95 \% \mathrm{CI})$. Backward stepwise logistic regression was performed to identify the best predictive model of service needs. Positive predictive value (PPV), sensitivity and specificity were calculated to identify whether health perception could be used to identify people in need of physical rehabilitation services.

142 seniors agreed to answer the survey yielding a response rate of $73 \%$. Among the respondents (mean age $79 \pm 7 ; 60 \%$ women), $40 \%$ rated their health as fair or poor. Seniors perceiving their health as fair or poor had higher odds of reporting impairments, activity limitations, and participation restricitions (OR ranging from 2.37; 95\% CI 1.03-5-45 to OR $12.22 ; 95 \%$ CI $2.68-55.78$ ) in comparison to those perceiving their health as good or better. The most significant predictors of service needs were community ambulation, household tasks, fatigue, and pain with $92 \%$ sensitivity and a maximum adjusted R-squared of 0.65 . Self-rated health used as single-item showed a positive predictive value (PPV) of 1 , sensitivity of $52 \%$, and specificity of $100 \%$. 